\title{
Microarray Analysis of Gene Expression Changes in Neuroplastin 65-Knockout Mice: Implications for Abnormal Cognition and Emotional Disorders
}

\author{
Huanhuan $\mathrm{Li}^{1} \cdot$ Jiujiang Zeng ${ }^{1} \cdot$ Liang Huang $^{1} \cdot$ Dandan Wu$^{1} \cdot$ Lifen Liu $^{1}$ • \\ Yutong Liu $^{2} \cdot$ Qionglan Yuan ${ }^{1}$
}

Received: 26 December 2017/ Accepted: 26 April 2018/Published online: 5 July 2018

(C) The Author(s) 2018

\begin{abstract}
Neuroplastin 65 (Np65) is an immunoglobulin superfamily cell adhesion molecule involved in synaptic formation and plasticity. Our recent study showed that Np65-knockout (KO) mice exhibit abnormal cognition and emotional disorders. However, the underlying mechanisms remain unclear. In this study, we found 588 differentiallyexpressed genes in Np65-KO mice by microarray analysis. RT-PCR analysis also revealed the altered expression of genes associated with development and synaptic structure, such as Cdhl, Htr3a, and Kcnj9. In addition, the expression of Wnt-3, a Wnt protein involved in development, was decreased in $N p 65-\mathrm{KO}$ mice as evidenced by western blotting. Surprisingly, MRI and DAPI staining showed a significant reduction in the lateral ventricular volume of Np65-KO mice. Together, these findings suggest that ablation of Np65 influences gene expression, which may contribute to abnormal brain development. These results provide clues to the mechanisms underlying the altered brain functions of Np65-deficient mice.
\end{abstract}

Keywords Neuroplastin $65 \cdot$ Microarray analysis $\cdot$ Gene expression profile $\cdot \mathrm{Htr} 3 \mathrm{a} \cdot \mathrm{Wnt}$

Huanhuan Li, Jiujiang Zeng and Liang Huang have contributed equally to this work.

Electronic supplementary material The online version of this article (https://doi.org/10.1007/s12264-018-0251-5) contains supplementary material, which is available to authorized users.

Qionglan Yuan

yqiongl@tongji.edu.cn

1 Department of Neurology, Shanghai Tongji Hospital, Tongji University School of Medicine, Shanghai 200065, China

2 Department of Radiology, University of Nebraska Medical Center, Omaha, NE 68198, USA

\section{Introduction}

Neuroplastin (Np) is a member of the immunoglobulin (Ig) superfamily of cell adhesion molecules and exists in two isoforms, Np65 and Np55 [1]. Np65 contains extracellular Ig1-2-3 modules, while Np55 only contains extracellular Ig2-3. Thus, Np65 can be differentiated from Np55 by its extracellular Ig1. Np55 is expressed in various organs and cell types, whereas the expression of Np65 is brain-specific and restricted to neurons.

Np65 undergoes trans- and cis-homophilic bindings as well as several heterophilic bindings with fibroblast growth factor receptors, the $\alpha 1$ or $\alpha 2$ subunit of $\mathrm{GABA}_{\mathrm{A}}$ receptors, and the basigin-monocarboxylate transporter [2-4]. Np65 has been implicated in the regulation of synaptic plasticity and the maintenance of excitatory/inhibitory balance. Antibodies specific for Np65 or recombinant Np65 block long-term potentiation (LTP) in the hippocampal CA1. The induction of LTP also increases the expression of Np65 in postsynaptic densities [5]. In addition, Nptn-deficient neurons exhibit impaired inhibitory transmission [6].

Previous studies have suggested that Np65 is associated with cognition and emotional states. Polymorphisms in the human NPTN gene have been shown to correlate with cortical thickness and intellectual abilities in adolescents as well as in patients with schizophrenia [7, 8]. Nptn-deficient mice exhibit retrograde amnesia, depressive-like behaviors, and decreased social interactions [9]. In addition, mutation of the Nptn gene results in deafness in mice, suggesting that NPTN is a novel deafness gene $[10,11]$. We have previously demonstrated that Np65 knock-out (KO) mice exhibit enhanced hippocampal-dependent spatial memory in the Morris water maze and step-through passive avoidance tests [12], but the underlying mechanisms were unclear. In this study, we used custom-designed microarray 
analysis to profile differentially-expressed genes in $\mathrm{Np} 65$ $\mathrm{KO}$ mice, in order to explain the altered brain functions in Np65-deficient mice.

\section{Materials and Methods}

\section{Animals}

The homozygous Np65-KO mice were obtained from engineered mouse models; this caused Np65-Ig1 deficiency in single chromosome as previously described [12]. Wildtype (WT) littermates served as controls. Animals were housed in a temperature-controlled environment under a 12 h light/dark cycle (08:00-20:00) with food and water ad libitum. All protocols complied with the National Institutes of Health Guide for the Care and Use of Laboratory Animals, and were approved by the Institutional Ethics Committee of Tongji University School of Medicine, and conformed to Directive 2010/63/EU and NIH guidelines.

\section{Microarray Experiments}

Microarray analysis was performed as previously described [13]. Briefly, animals were sacrificed after deep anesthesia with intraperitoneal (i.p.) injection of $1 \%$ pentobarbital sodium $(30 \mathrm{mg} / \mathrm{kg})$. Hippocampi from adult Np65-KO mice ( 3 months old) and age-matched WT mice $(n=$ 3/genotype) were dissected and immediately frozen in liquid nitrogen. The samples were stored at $-80^{\circ} \mathrm{C}$ until use.

Total RNA was extracted from the hippocampal tissue using TRIzol (15596026, Thermo Fisher Scientific, Waltham, MA) and further purified with an RNeasy Mini Kit (74104, Qiagen, Hilden, Germany). RNA concentration and quality were evaluated by spectrophotometry (NanoDrop ND-1000, Thermo Fisher Scientific, Waltham, MA). One microgram of total RNA was amplified and labeled with a One-Color Quick Amp Labeling Kit (5190-0442, Agilent Technologies, Santa Clara, CA). The fluorescencelabeled cRNA was hybridized onto the Whole Mouse Genome Oligo Microarray $(4 \times 44 \mathrm{~K}$, Agilent Technologies, Santa Clara, CA) using the Agilent Gene Expression Hybridization Kit (5188-5242, Agilent Technologies, Santa Clara, CA). Chips were washed and scanned by a microarray scanner (G2565BA, Agilent Technologies, Santa Clara, CA). Raw data were then normalized and analyzed using the GeneSpring GX Software Package (v11.5, Agilent Technologies). The microarray experiment was performed with 3 biological and experimental repeats. Normalized values were used to screen for differentiallyexpressed genes from biological and experimental repeats before all replicates were combined. Genes with a foldchange of $>2.0$ and a $P$ value $<0.05$ were selected for Gene Ontology (GO) and pathway analysis.

\section{Gene Ontology and Pathway Analysis}

The fold-changes of differential expression were determined by the abundance ratio of $N p 65-\mathrm{KO}$ and WT mice. Hierarchical clustering was used to analyze the differentially-expressed genes. GO analysis was applied to analyze the cellular components, biological functions, and biological processes of the differentially-expressed genes (www. geneontology.org). Pathway analysis was used to reveal significant Kyoto Encyclopedia of Genes and Genomes (KEGG) pathways of the differentially-expressed genes.

\section{Quantitative Real-Time Reverse-Transcription PCR}

Quantitative real-time reverse-transcription PCR (RTPCR) was performed as previously described [14]. Adult Np65-KO and WT mice ( $n=4 /$ genotype) were sacrificed after anesthesia with $1 \%$ pentobarbital sodium $(30 \mathrm{mg} / \mathrm{kg}$, i.p.) and the forebrain was harvested to extract total RNA using TRIzol (15596026, Thermo Fisher Scientific, Waltham, MA). RNA concentration and quality were determined by NanoDrop (ND-1000, Thermo Fisher Scientific, Waltham, MA). cDNA was generated using reverse transcriptase (PrimeScript ${ }^{\mathrm{TM}}$ RT reagent Kit, RR0747Q, Takara Bio, Tokyo, Japan). The first-strand cDNA was used as a template for RT-PCR analysis. The primers for RT-PCR analysis (Table 1) were designed by the NCBI primer designing tool [15] and synthesized by Sangon (Shanghai, China). Each RT-PCR reaction was carried out in a $20 \mu \mathrm{L}$ volume using SYBR Green Master Mix (RR820Q, Takara Bio, Tokyo, Japan), started at $30 \mathrm{~s}$ at $95^{\circ} \mathrm{C}$ for initial denaturation, followed by 40 cycles of $5 \mathrm{~s}$ at $95^{\circ} \mathrm{C}$ and $34 \mathrm{~s}$ at $60^{\circ} \mathrm{C}$ in the ABI 7500 Real-Time PCR System. A total of 3 independent samples per subject were run in duplicate for RT-PCR. $\beta$-actin was used as the reference gene. The $2^{-\Delta \Delta \mathrm{Ct}}$ method was used to determine the relative expression levels of genes.

\section{Western Blotting}

Adult Np65-KO and WT mice (4 months old, $n=$ 3/genotype) were used for western blotting. Briefly, animals were decapitated after deep anesthesia with $1 \%$ pentobarbital sodium $(30 \mathrm{mg} / \mathrm{kg}$ i.p.). Forebrains were collected and frozen in nitrogen and then stored at $-80^{\circ} \mathrm{C}$ until use. The total proteins were extracted using RIPA lysis buffer (P0013B, Beyotime) with $1 \mathrm{mmol} / \mathrm{L}$ PMSF (ST506, Beyotime). Protein concentrations were measured using the BCA Protein Assay Kit (P0010, Beyotime, 
Table 1 Primers used in RTPCR.

\begin{tabular}{llll}
\hline Gene & NCBI Accession & Forward Primer & Reverse Primer \\
\hline Cdh1 & NM_009864 & CAGCCGGTCTTTGAGGGATT & TGACGATGGTGTAGGCGATG \\
Cdh4 & NM_009867 & ACAACCGTCCCGAGTTCATC & TCATCTGCATCGTTGGCTGT \\
Htr3a & NM_013561 & CAGACCACCTCCTGGCTAAC & GATGCTGTCTGTGGGGATGG \\
Htr4 & NM_008313 & ACGTCCTCATGCCCATTTCC & ACCACTGCAAGGAACGTGAG \\
Kcnj9 & NM_008429 & TCTTCTTCGTGCTCGCCTAC & CGAAGCCGTTGAGGTTGTTG \\
Pla2g4e & NM_177845 & CTCCAACTGCCTACACCCAG & CCTCTGGGTTGAGTGGGAAC \\
Xaf1 & NM_001037713 & AGAGCCCATCCCAGAGTCAA & CAGATTGCTAAGCTGCACGG \\
Lactb & NM_030717 & GGCTATGCAGACGTGGAGAA & CAGTTTAGCCAGAGCCACCA \\
Actb & NM_007393 & GCTGTATTCCCCTCCATCGTG & AGTCCTTCTGACCCATTCCCA \\
\hline
\end{tabular}

Jiangsu, China), then $10 \mathrm{ng}$ of total protein was separated by SDS-PAGE and transferred to the PVDF membrane. After blocking with $5 \%$ bovine serum albumin (BSA), the membranes were incubated overnight at $4{ }^{\circ} \mathrm{C}$ with primary antibody against Wnt-3 (1:1,000, Santa Cruz Biotechnology, Dallas, TX) and mouse anti-glyceraldehyde-3-phosphate dehydrogenase (GAPDH, 1:1,000, Santa Cruz Biotechnology, Dallas, TX). Subsequently, the membranes were incubated with HRP-conjugated goat anti-mouse secondary antibody $(1: 1,000$, Beyotime) for $2 \mathrm{~h}$ at room temperature. The labeled proteins were detected by using the ImageQuant LAS 4000mini system (GE Healthcare Life Sciences, Chicago, IL). The protein levels were normalized to that of GAPDH from three independent experiments.

\section{Magnetic Resonance Imaging (MRI)}

A separate cohort of mice (4 months old, $n=5 /$ genotype) was used in MRI analysis. MRI data were acquired with a 7.0 T animal MRI scanner (PharmaScan, Bruker Biospin GmbH, Germany) with 4-channel phased array coil. T2weighed (T2-wt) MRI was performed using a rapid acquisition with relaxation enhancement (RARE) sequence with TR/TE $=4200 / 36 \mathrm{~ms}$, RARE factor $=8$, and averaging number $=3$. The geometric parameters for the scan were: slice number $=18$, slice thickness $=0.5 \mathrm{~mm}$, matrix $=256 \times 256$, and FOV $=21 \times 21 \mathrm{~mm}^{2}$.

\section{4',6-Diamidino-2-Phenylindole (DAPI) Staining}

Adult Np65-KO and WT mice ( $n=4,2$ months old) were anesthetized with $1 \%$ pentobarbital sodium intraperitoneally and perfused with $4 \%$ paraformaldehyde. The brain was removed, postfixed for $10 \mathrm{~h}-16 \mathrm{~h}$, and cryoprotected in $20 \%$ sucrose. Coronal sections $(10 \mu \mathrm{m}$, at the level of the lateral ventricle, $0 \mathrm{~mm}-2 \mathrm{~mm}$ from bregma) were prepared for DAPI staining. In brief, the sections were blocked in 5\% BSA (B2064-100G, Sigma-Aldrich, St. Louis, MO) with $0.3 \%$ Triton X-100 (ST795,
Beyotime), then incubated with DAPI (1:300, Beyotime) diluted in $1 \%$ BSA with $0.3 \%$ Triton $\mathrm{X}-100$ for $10 \mathrm{~min}$ at room temperature. The sections were then rinsed with PBS and covered with Permount for fluorescent microscopy (Eclipse 80i, Nikon Corp., Tokyo, Japan).

\section{Statistics}

Statistics were calculated using SPSS Statistics software (v22.0, IBM). All data are presented as mean \pm SEM. Independent samples were tested by the unpaired Student's $t$-test (two-tailed). The Mann-Whitney $U$ test was used to determine the significance of data with an abnormal distribution or unequal variance. Statistical significance was set at $P<0.05$.

\section{Results}

\section{Microarray Analysis of Differentially-Expressed Genes in the Hippocampus of Np65-KO Mice}

All genes are shown in a scatter plot with normalized intensity in Fig. 1A (details in Table S1). Of the 34397 targeted genes by the Mouse $4 \times 44 \mathrm{~K}$ Gene Chip, 481 $(1.4 \%)$ were up-regulated and $418(1.2 \%)$ were downregulated by 2 -fold in the Np65-KO mice (Fig. 1B). Using $P<0.05$ as the criterion, 367 genes were significantly higher and 221 genes were significantly lower in $\mathrm{Np} 65-\mathrm{KO}$ mice as compared to age-matched WT mice (Fig. 1C and D, Table S2). These differentially-expressed genes were primarily located on chromosomes 7, 9 and 11. Notably, the NPTN gene resides on chromosome 9 (Fig. 1E).

\section{Gene Ontology and Pathway Analysis of Differen- tially-Expressed Genes}

Using the criterion of $P<0.001$, GO analysis showed that the upregulated genes were involved in several cellular components, including extracellular region, plasma 
A

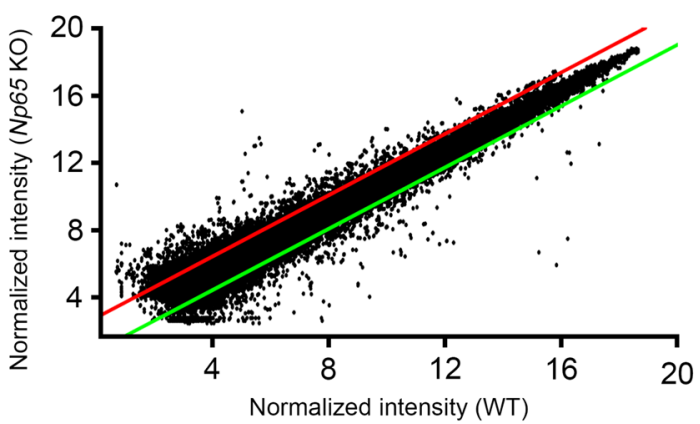

B

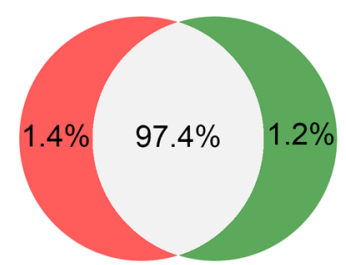

C

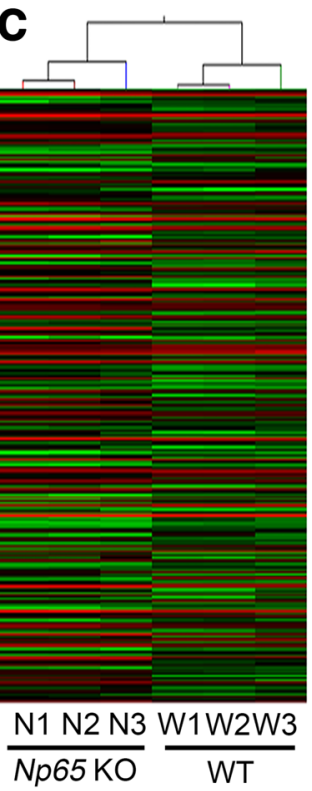

D $80, \bullet$ up

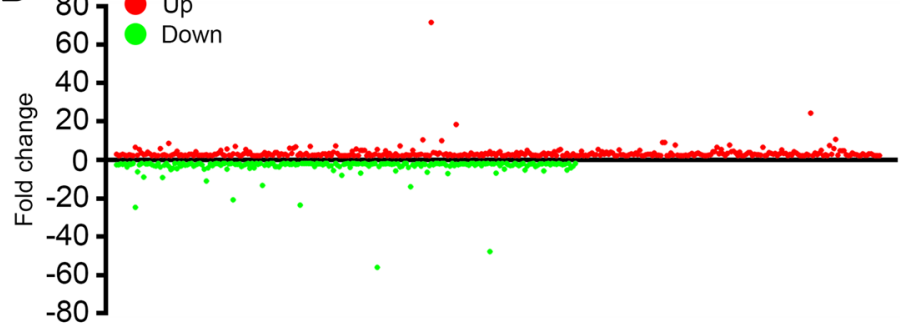

$\mathbf{E}$

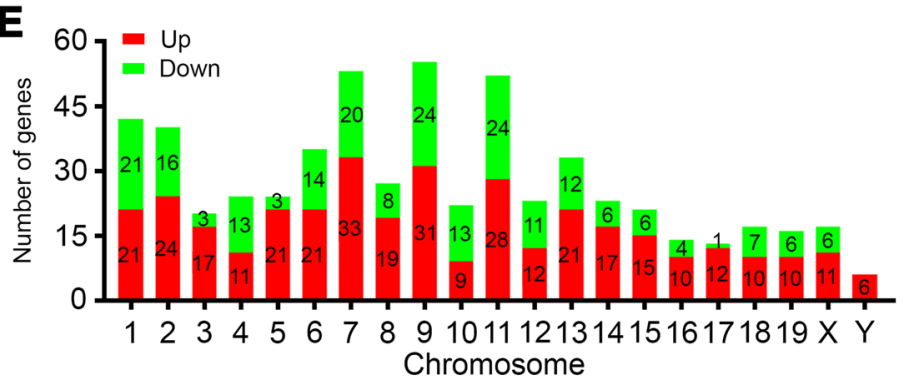

Fig. 1 Differentially-expressed genes in the hippocampus of Np65-KO mice. A Scatter plot of normalized intensity derived from microarray chips with WT and Np65-KO mice. Dots above the red line denote upregulated genes, and dots below the green line denote downregulated genes. B Venn diagram showing the percentages of differentially-expressed genes categorized by fold-change. C Hierarchical clustering of differentially-expressed genes. N1-N3, Np65-KO mice; W1-W3, WT mice. D Fold changes of differentially-expressed genes in Np65-KO mice. E Chromosome distributions of differentially-expressed genes in Np65-KO mice. Red bars, numbers of upregulated genes; green bars, numbers of downregulated genes.

membrane, and desmosome (Fig. 2A). The molecular functions of the upregulated genes were mainly associated with co-receptor activity. The down-regulated genes were involved in various binding actions, such as ankyrin binding, lipoprotein binding, and Wnt-protein binding (Fig. 2B). The main biological processes were the cellular response to interferon-beta, embryonic hindlimb morphogenesis, negative regulation of neuron differentiation, cellular process, positive regulation of epidermis development, and negative regulation of transmembrane receptor protein serine/threonine kinase signaling pathway. The downregulated genes were associated with several biological processes, including post-transcriptional regulation of gene expression, developmental growth involved in morphogenesis, regulation of translation, cell adhesion, developmental growth, and response to stimuli (Fig. 2C).
According to the pathway analysis, 8 pathways were significantly up-regulated in $\mathrm{Np} 65-\mathrm{KO}$ mice, including glycerophospholipid metabolism, pancreatic secretion, and regulation of actin cytoskeleton. Among the 9 downregulated pathways, the most prominent was cytokinecytokine receptor interaction. The other downregulated pathways were involved in bladder cancer, chemical carcinogenesis, and drug metabolism (Fig. 2D).

\section{Functional Analysis of Differentially-Expressed Genes in Np65-KO Mice}

The differentially-expressed genes were divided into four categories: cell adhesion, development, neurotransmission and ion channel, and signal transduction. Previous studies have suggested that Np65 may interact with other cell 
A

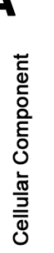

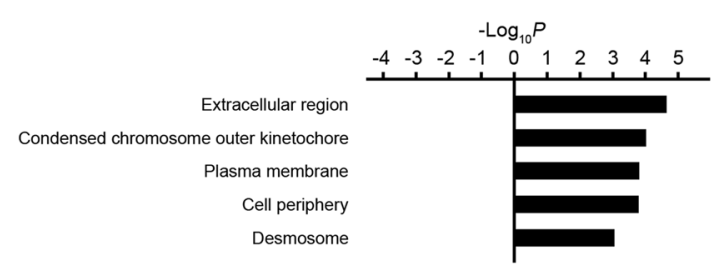

C
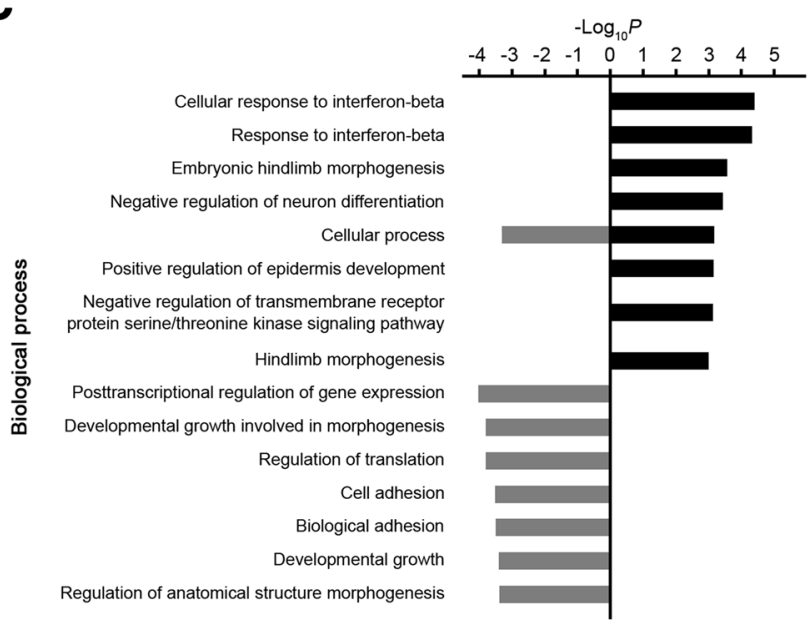

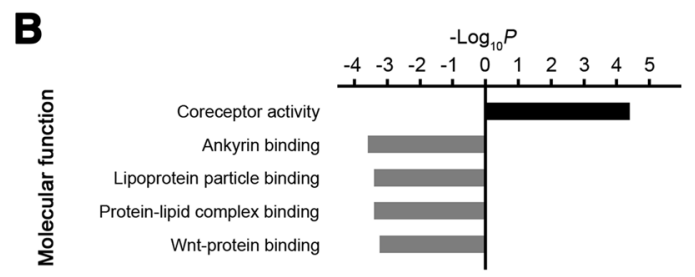

D

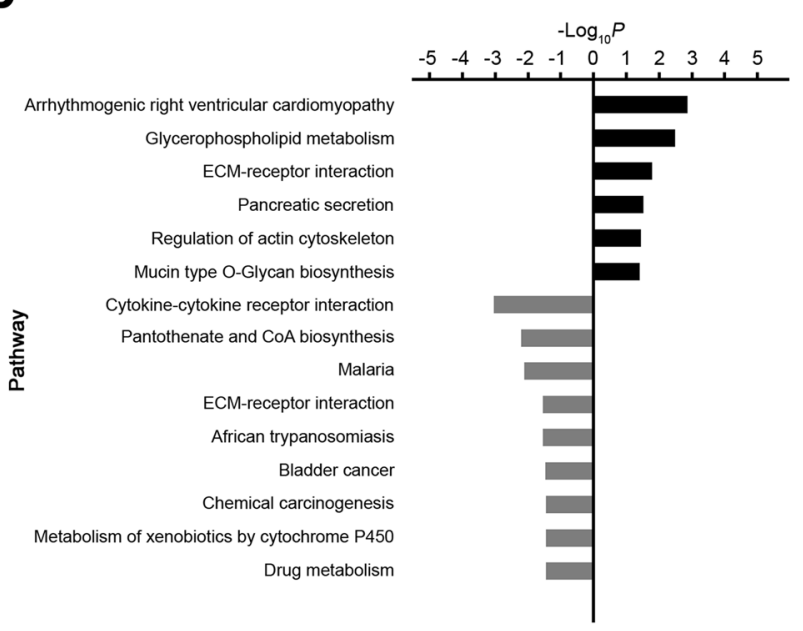

Fig. 2 GO and pathway analysis of differentially-expressed genes in Np65-KO mice. A-C Cellular components (A), molecular functions (B), and biological processes $(\mathbf{C})$ of differentially-expressed genes. D Significantly changed pathways in Np65-KO mice.

adhesion molecules like fibroblast growth factor receptor (FGFR) to activate intracellular signaling [2]. Interestingly, the expression of several cell adhesion molecules was altered in Np65-KO mice. Fgfr4, immunoglobulin superfamily member 1 (Igsfl), interleukin 7 receptor (Il7r), and members of the integrin superfamily such as integrin alpha 1 (Itgal) and integrin alpha 9 (Itga9) were upregulated, while interleukin 1 receptor, type II (IIIr2) and protein tyrosine phosphatase receptor type D (Ptprd) were down-regulated in Np65-KO mice. In Np65-KO mice, the expression levels of genes associated with $\mathrm{Ca}^{2+}$ binding, including $\mathrm{Ca}^{2+}$-binding protein 5 (Cabp5), calbindin 1 (Calb1), and calmodulin-like 4 (Calml4) were significantly increased. However, several cadherins, including cadherin $1(C d h 1)$, cadherin $4(C d h 4)$, cadherin $6(C d h 6)$, protocadherin $7(P c d h 7)$, protocadherin 12 (Pcdh12), and protocadherin 17 (Pchd17) were downregulated in $\mathrm{Np} 65$-KO mice (Table 2).

In addition, a subset of genes associated with neuronal development, such as chemokine (C-C motif) receptor 5 (Ccr5), forkhead box O3 (Foxo3), myelin basic protein $(M b p)$, myocyte enhance factor $2 \mathrm{C}(M e f 2 c)$, and Wnt inhibitory factor 1 (Wifl), were significantly downregulated in $\mathrm{Np} 65-\mathrm{KO}$ mice, while several genes involved in glial cell development, including neurotrophin 3 (Ntf3), glial cell missing homolog $1(\mathrm{Gcml})$, and transformation related protein 73 (Trp73), were upregulated. More interestingly, the expression of eye development-related genes was also altered in Np65-KO mice. The expression of aldehyde dehydrogenase family 1 subfamily A3 (Aldhla3), retinitis pigmentosa GTPase regulator interacting protein 1 (Rpgripl), crumbs homolog 1 (Crbl), visual system homeobox 1 homolog $(V s x l)$, keratin 12 (Krt12), and secreted frizzled-related sequence protein 5 (Sfrp5) were down-regulated. Myosin VIIA (Myo7a) and collagen triple helix repeat containing 1 (Cthrcl), which are associated with inner ear receptor cell development, were also decreased in Np65-KO mice (Table 2).

The expressions of genes related to the structure and function of synapses were also altered in Np65-KO mice. Notably, expression of the serotonin receptor 4 (Htr4) gene was significantly increased, whereas the expression of serotonin receptor $3 \mathrm{~A}(\mathrm{Htr} 3 \mathrm{a})$ was significantly decreased in Np65-KO mice. Moreover, two ion channel-related genes displayed significant downregulations in Np65-KO mice: $\mathrm{Cl}^{-}$channel $\mathrm{Ca}^{2+}$ activated 5 (Clca5) and $\mathrm{K}^{+}$inwardlyrectifying channel subfamily J member 9 (Kcnj9) (Table 2).

MAPK signaling is essential for various physiological and pathological processes, such as neural plasticity and memory. We found that the expression of mitogenactivated protein kinase kinase 7 (Map2k7) was significantly decreased, while phospholipase A2, group IVE (Pla2g4e), serine/threonine kinase 38 like (Stk38l), and 
Table 2 Selected differentially-expressed genes in the hippocampus of Np65-KO mice.

\begin{tabular}{|c|c|c|c|c|}
\hline Category & NCBI accession & Full name & Fold change & $P$ value \\
\hline \multicolumn{5}{|c|}{ Cell adhesion } \\
\hline$F g f r 4$ & NM_008011 & Fibroblast growth factor receptor 4 & 2.0 & 0.004 \\
\hline Igsf1 & NM_177591 & Immunoglobulin superfamily, member 1 & 2.3 & 0.002 \\
\hline$I l 7 r$ & NM_008372 & Interleukin 7 receptor & 2.1 & 0.006 \\
\hline$I l 1 r 2$ & NM_010555 & Interleukin 1 receptor, type II & -2.1 & 0.002 \\
\hline Ptprd & XR_107615 & Protein tyrosine phosphatase, receptor type D & -2.3 & 0.015 \\
\hline$C d h 1$ & NM_009864 & Cadherin 1 & -2.4 & 0.010 \\
\hline$C d h 4$ & NM_009867 & Cadherin 4 & -2.1 & 0.004 \\
\hline Cdh6 & NM_007666 & Cadherin 6 & -2.2 & 0.043 \\
\hline Pcdh7 & NM_001122758 & Protocadherin 7 & -5.8 & 0.020 \\
\hline Pcdh12 & NM_017378 & Protocadherin 12 & -2.3 & 0.045 \\
\hline Pcdh17 & NM_001013753 & Protocadherin 17 & -2.2 & 0.000 \\
\hline Itgal & NM_001033228 & Integrin alpha 1 & 3.0 & 0.047 \\
\hline Itga9 & NM_133721 & Integrin alpha 9 & 10.4 & 0.000 \\
\hline \multicolumn{5}{|c|}{ Development } \\
\hline Ccr5 & NM_009917 & Chemokine (C-C motif) receptor 5 & -2.1 & 0.009 \\
\hline Foxo3 & AK143198 & Forkhead box O3 & -2.0 & 0.004 \\
\hline$M b p$ & NM_010777 & Myelin basic protein & -2.2 & 0.014 \\
\hline $\operatorname{Mef} 2 c$ & NM_025282 & Myocyte enhancer factor $2 \mathrm{C}$ & -2.1 & 0.034 \\
\hline Wif1 & NM_011915 & Wnt inhibitory factor 1 & -2.1 & 0.001 \\
\hline$N t f 3$ & NM_001164034 & Neurotrophin 3 & 3.2 & 0.044 \\
\hline Gcml & NM_008103 & Glial cells missing homolog 1 & 2.9 & 0.019 \\
\hline $\operatorname{Trp} 73$ & NM_011642 & Transformation related protein 73 & 2.3 & 0.040 \\
\hline Aldhla3 & NM_053080 & Aldehyde dehydrogenase family 1 , subfamily A3 & -2.1 & 0.008 \\
\hline Rpgripl & NM_023879 & Retinitis pigmentosa GTPase regulator interacting protein 1 & -2.5 & 0.020 \\
\hline Crbl & NM_133239 & Crumbs homolog 1 & -2.5 & 0.039 \\
\hline Vsxl & NM_054068 & Visual system homeobox 1 homolog & -3.4 & 0.015 \\
\hline Krt12 & NM_010661 & Keratin 12 & -2.9 & 0.000 \\
\hline Sfrp5 & NM_018780 & Secreted frizzled-related sequence protein 5 & -2.0 & 0.006 \\
\hline Myo7a & NM_008663 & Myosin VIIA & -2.8 & 0.040 \\
\hline Cthrcl & NM_026778 & Collagen triple helix repeat containing 1 & -2.1 & 0.000 \\
\hline \multicolumn{5}{|c|}{ Neurotransmission and ion channel } \\
\hline Cabp5 & NM_013877 & $\mathrm{Ca}^{2+}$ binding protein 5 & 3.5 & 0.031 \\
\hline Calb1 & AK038856 & Calbindin 1 & 2.1 & 0.004 \\
\hline Calml4 & NM_138304 & Calmodulin-like 4 & 3.0 & 0.007 \\
\hline Cplx2 & NM_009946 & Complexin 2 & 2.1 & 0.033 \\
\hline Htr4 & NM_008313 & 5-hydroxytryptamine (serotonin) receptor 4 & 2.4 & 0.043 \\
\hline$H t r 3 a$ & NM_013561 & 5-hydroxytryptamine (serotonin) receptor $3 \mathrm{~A}$ & -2.5 & 0.001 \\
\hline Clca5 & NM_178697 & $\mathrm{Cl}^{-}$channel $\mathrm{Ca}^{2+}$ activated 5 & 2.9 & 0.000 \\
\hline Kcnj9 & NM_008429 & $\mathrm{K}^{+}$inwardly-rectifying channel, subfamily J, member 9 & 4.2 & 0.000 \\
\hline \multicolumn{5}{|c|}{ Signal transduction } \\
\hline Map $2 k 7$ & NM_001042557 & Mitogen-activated protein kinase kinase 7 & -2.67 & 0.000 \\
\hline Pla2g4e & NM_177845 & Phospholipase A2, group IVE & 4.64 & 0.000 \\
\hline Stk38l & NM_172734 & Serine/threonine kinase 38 like & 4.80 & 0.013 \\
\hline Ppp6rl & NM_172894 & Protein phosphatase 6 , regulatory subunit 1 & 2.95 & 0.033 \\
\hline Xaf1 & NM_001037713 & XIAP associated factor 1 & 5.99 & 0.000 \\
\hline Lactb & NM_030717 & Lactamase, beta & 24.30 & 0.000 \\
\hline
\end{tabular}



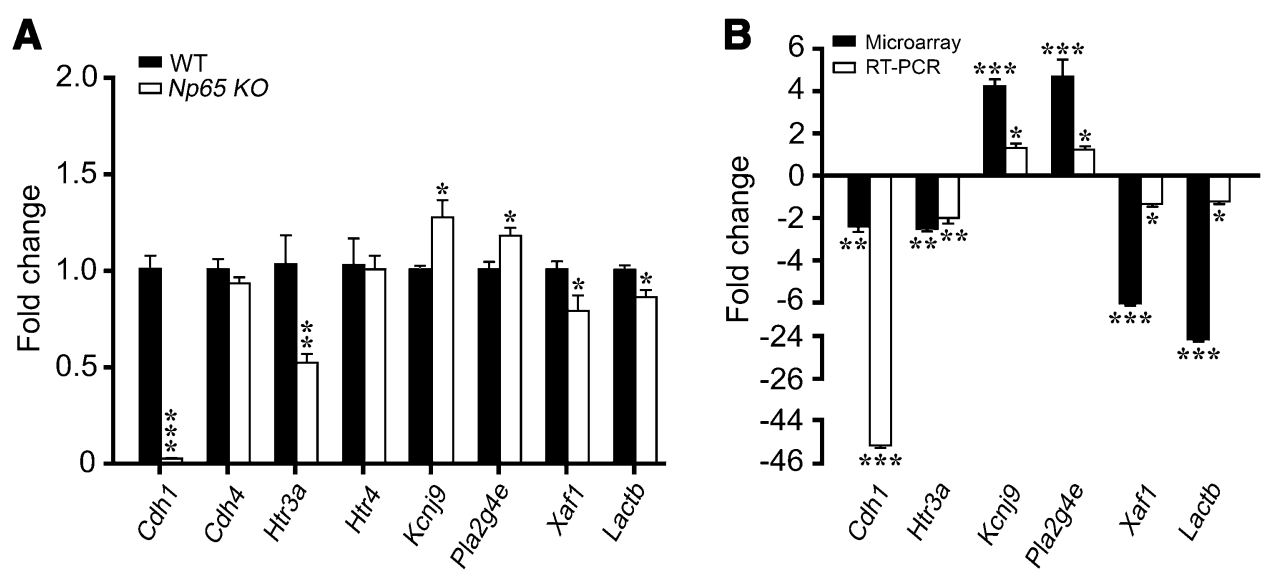

Fig. 3 Relative expression levels of selected genes in Np65-KO and WT mice. A Results of quantitative real-time PCR (RT-PCR) $(n=4$ mice). B RT-PCR and microarray experimental results for relative gene expression in $\mathrm{Np} 65-\mathrm{KO}$ and WT mice. The relative expression levels were calculated as the ratio of the target gene expression level to the $\beta$-actin expression level in the same sample. Fold changes are shown as mean \pm SEM. $* P<0.05, * * P<0.01, * * * P<0.001$.

Fig. 4 Reduced expression of Wnt-3 in Np65-KO mice.

A Representative Wnt-3 bands from the forebrain of WT $(n=$ 5) and Np65-KO mice $(n=6)$. B Quantitative results of western blotting analysis showed that the expression level of Wnt3 was significantly decreased in the forebrain of $\mathrm{Np} 65-\mathrm{KO}$ mice. All data are presented as mean \pm SEM. $* * P<0.01$.
A

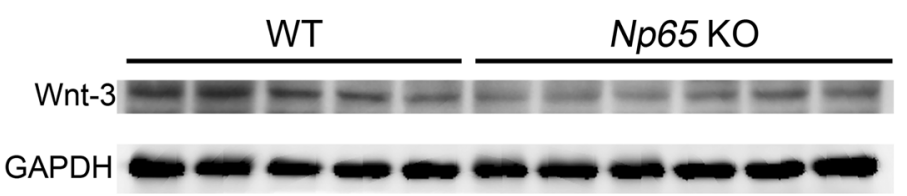

B

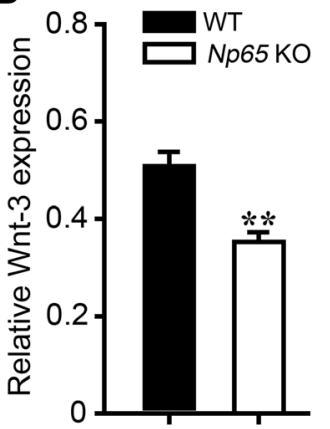

protein phosphatase 6 , regulatory subunit 1 (Ppp6rl) were significantly increased in Np65-KO mice. In addition, the expression level of $\mathrm{X}$-linked inhibitor of apoptosis protein associated factor 1 (Xafl), an apoptosis-promoting factor, was decreased. Most notably, $\beta$-lactamase (Lactb), which is involved in mitochondrial metabolism, was also significantly down-regulated in Np65-KO mice (24.30-fold, $P<0.001$ ) (Table 2).

\section{RT-PCR Analysis of Differentially-Expressed Genes}

Np65 is highly expressed in the hippocampus and other brain regions, such as cortex and striatum [1]. Therefore, 8 genes related to the functions of $\mathrm{Np} 65$ were further selected for RT-PCR analysis. The results showed that 6 of these were also significantly changed in the forebrain of Np65KO mice (Fig. 3A), including the downregulated Cdh1 (fold change, 45.34, $P<0.001$ ), Htr3a (fold change, 1.94, $P<0.01$ ), Xafl and Lactb, and the increased Kcnj9 (fold change, 1.26, $P<0.05$ ) and Pla2g4e (fold change, 1.17, $P<0.05$ ) (Fig. 3B).

\section{Decreased Expression of Wnt-3 in Np65-KO Mice}

Microarray and RT-PCR analysis showed that the expression levels of several genes associated with development were altered in Np65-KO mice. Some differentiallyexpressed genes, such as Wifl and $C d h 1$, are involved in Wnt signaling. Wnt signaling is a crucial regulator of many developmental processes, such as cell proliferation, maintenance of stem cells, and cell fate determination [16]. Therefore, we examined the protein level of Wnt-3 in Np65-KO mice by western blotting. The results showed that the protein level of Wnt-3 was significantly lower in the forebrain of Np65-KO mice than in WT mice (Fig. 4).

\section{Reduced Lateral Ventricles in Np65-KO Mice}

Given that the dysfunction of Wnt signaling may influence brain development, we then assessed whether ablation of Np65 affects the brain morphology of mice. T2-wt images were used to assess region-specific volume changes. The gross brain architecture was not affected in Np65-KO mice. 
A
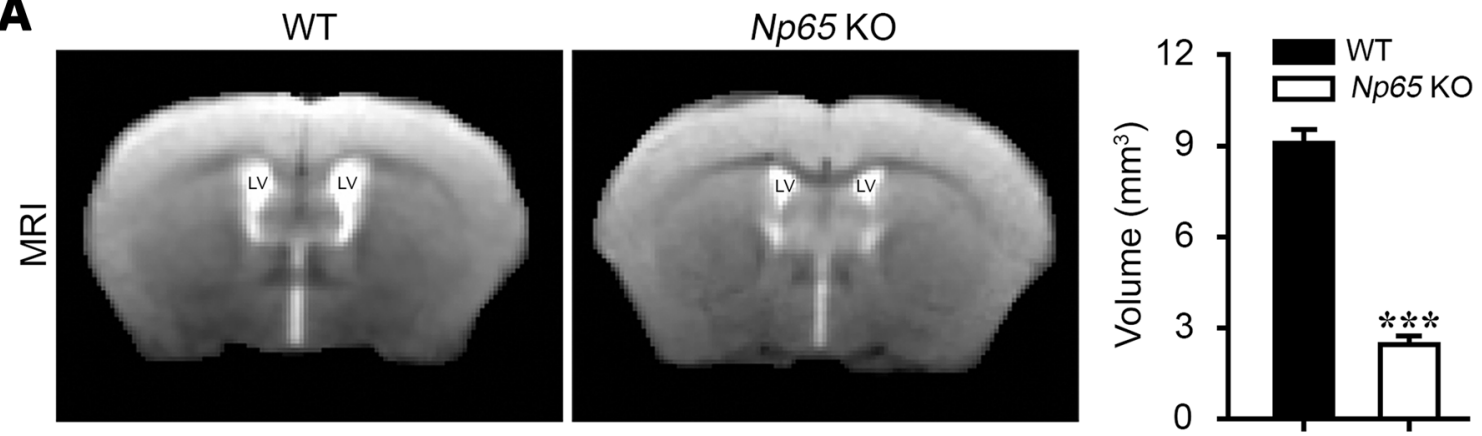

B
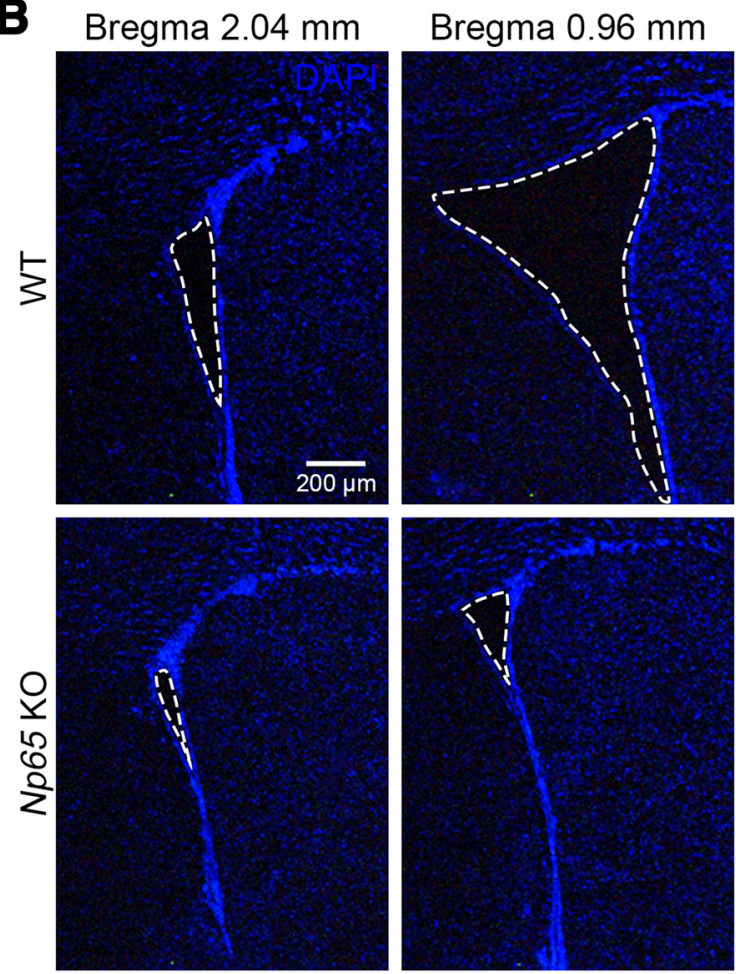

Fig. 5 Reduction in lateral ventricles in Np65-KO mice. A T2-wt MRI showing a significant reduction in the lateral ventricles (LV) compared to WT mice. B DAPI staining showing a significant

MRI morphometry also showed normal anatomy of the cerebral cortex, hippocampus, thalamus, hypothalamus, basal ganglia, and caudatoputamen of Np65-KO mice. However, the lateral ventricular volume was significantly reduced compared to WT mice (Fig. 5A), and this was further confirmed by DAPI staining (Fig. 5B). Thus, these results suggested that the absence of $\mathrm{Np} 65$ leads to altered architecture of the mouse brain.

\section{Discussion}

Np65 is specifically expressed in the brain and has been reported to mediate several cellular processes including cellcell adhesion, neurite outgrowth, and synaptic plasticity
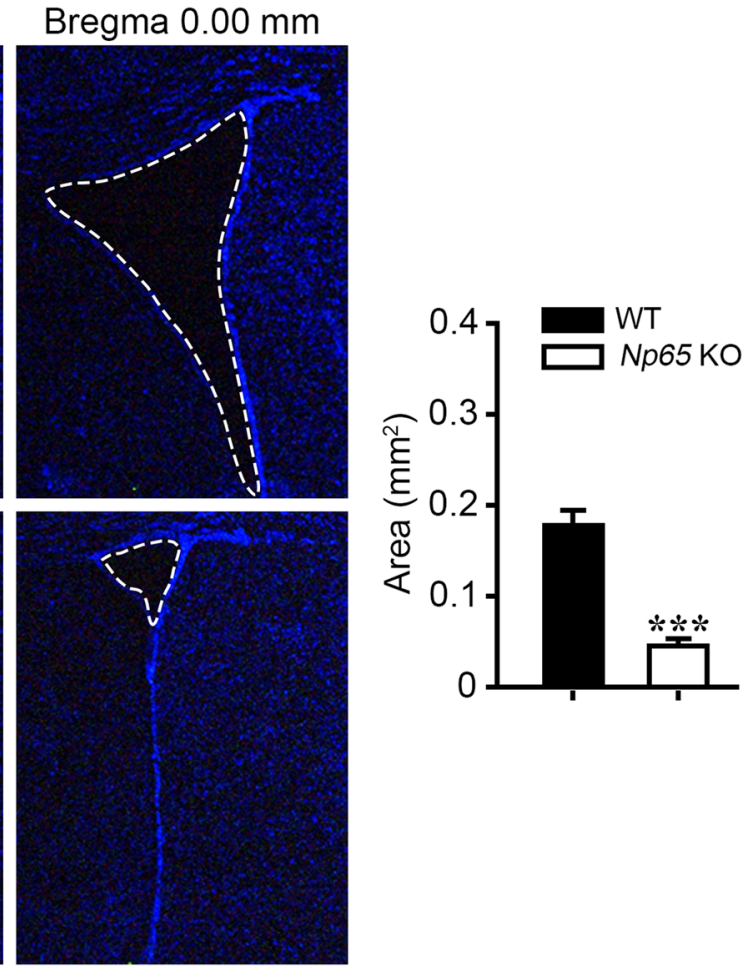

reduction in the lateral ventricles in coronal sections from adult $\mathrm{Np} 65$ KO mice compared to WT mice. Scale bar, $200 \mu \mathrm{m}$. ${ }^{* * *} P<0.001$.

$[2,5,17,18]$. Our previous studies have shown that $\mathrm{Np} 65$ KO mice exhibit abnormal cognitive and emotional behaviors [12]. To investigate the underlying mechanisms, we further analyzed the gene expression profiles in Np65-KO mice in this study. Our microarray analysis demonstrated a large number of differentially-expressed genes in Np65-KO mice; these genes are crucially involved in development, ion channels, neurotransmission, and signal transduction.

Our study identified many differentially-expressed genes involved in neuronal development, such as the decreased expressions of Cdh1, Ccr5, Foxo3, Mbp, Wifl, and Mef2c, as well as upregulation of $N t f 3, G c m l$, and $\operatorname{Trp} 73$, implying that Np65 deletion affects the configuration of the brain. Coincidently, T2-wt MRI morphometry and brain slices stained with DAPI showed a significant reduction in lateral 
ventricular volume in $\mathrm{Np} 65-\mathrm{KO}$ mice compared to WT mice. The expression of Wnt-3 was significantly decreased in Np65-KO mice. Wnt signaling is a crucial regulator of developmental processes like cell proliferation and cell fate determination [16]. Dysregulation of Wnt signaling may contribute to neuropsychiatric disorders, such as depression and schizophrenia [19]. In this study, our findings suggested that Np65 deletion affects the Wnt signaling pathway by decreasing Wnt expression. Together, these differentially-expressed genes associated with development may contribute, at least in part, to changes in the ventricles and abnormal behaviors in Np65-KO mice.

Recent studies have reported that mutation of the NPTN gene results in deafness in mice [10]. It has been reported that Np65 may regulate the properties of synapses connecting the inner hair cells with spiral ganglion neurons [10]. Intriguingly, Zeng et al. reported that Np55 is expressed in stereocilia of outer but not inner hair cells and affects interactions of stereocilia with the tectorial membrane and cochlear amplification in mice with NPTN mutation [11]. Together, these recent findings clearly confirm NPTN as a novel deafness gene. Consistent with their reports, our microarray analysis showed that the genes associated with inner ear receptor cell development, myosin VIIA (Myo7a) and collagen triple helix repeat containing 1 (Cthrcl) were significantly decreased in Np65-KO mice, supporting the hypothesis that Np65 is involved in hearing.

It has been shown that Np65 is linked with ribbon synapse formation in the plexiform layers of the rat retina [20]. Retinal function, as assessed using the electroretinogram, is unaffected by the absence of NPTN [10]. Surprisingly, the involvement of Np65 in vision was demonstrated using the pupillary light reflex and flash visual evoked potentials (our unpublished data). In agreement with our finding, our microarray analysis showed that the expression of eye development-related genes, including Aldh1a3, Rpgripl, Crb1, Vsxl, Krt12, and Sfrp5, was significantly downregulated in Np65-KO mice. Although these alterations in eye-development genes need to be confirmed, the reduced amplitude of the pupil in the pupillary light reflex and reduced first negative and positive amplitude of flash visual evoked potentials (our unpublished data) suggest that Np65 plays roles in vision.

Our previous studies have shown that Np65-KO mice appear to show enhanced memory in the Morris water maze and increased anxiety [12]. Central 5-hydroxytryptamine (5HT) activity is involved in emotional and cognitive activities [21, 22]. Generally, stimulation of central 5-HT activity impairs cognition, while its inhibition enhances cognition in rodent models. Tropisetron, a selective $5-\mathrm{HT}_{3}$ receptor antagonist, has been confirmed to reverse the cognitive deficit in rats injected with $\mathrm{A} \beta(1-42)$ [23]. In addition, central 5-HT activity is closely associated with anxiety [24-27]. Among the 5-HT receptors, 5- $\mathrm{HT}_{3}$ is the only ligand-gated ion channel that increases intracellular cations such as $\mathrm{Ca}^{2+}, \mathrm{Na}^{+}$, and $\mathrm{K}^{+}$. Stimulation of 5- $\mathrm{HT}_{3}$ receptors induces the rapid and transient depolarization of neurons. 5-HT receptor 3A-null mice exhibit anxiolytic behaviors, indicating that this receptor influences anxiety-like behavior [28]. More surprisingly, microarray and RT-PCR analysis demonstrated that Htr3a mRNA was significantly reduced in Np65-KO mice. How deletion of Np65 affects the expression of $H t r 3 a$ remains to be determined. To date, the decreased expression of $H$ tr $3 a$ may explain, at least in part, the changed cognitive and anxiety behaviors in $\mathrm{Np} 65$-KO mice.

In conclusion, the present study demonstrates that a large number of genes are differentially expressed in Np65KO mice. Notably, microarray analysis in Np65-KO mice revealed altered expression of $\mathrm{Htr} 3 \mathrm{a}$ and genes associated with development, hearing, and vision, which may provide important insights for understanding the role of Np65 in brain development as well as brain functions like cognition and emotion.

Acknowledgements This work was supported by the National Natural Science Foundation of China (81371213, 81070987, and 30971531) and the grants from the Ministry of Science and Technology of China (2010CB945600 and 2010CB945601).

\section{Compliance with Ethical Standards}

Conflict of interest All authors claim that there are no conflicts of interest.

Open Access This article is distributed under the terms of the Creative Commons Attribution 4.0 International License (http:// creativecommons.org/licenses/by/4.0/), which permits unrestricted use, distribution, and reproduction in any medium, provided you give appropriate credit to the original author(s) and the source, provide a link to the Creative Commons license, and indicate if changes were made.

\section{References}

1. Langnaese K, Beesley PW, Gundelfinger ED. Synaptic membrane glycoproteins gp65 and gp55 are new members of the immunoglobulin superfamily. J Biol Chem 1997, 272: 821-827.

2. Owczarek S, Soroka V, Kiryushko D, Larsen MH, Yuan Q, Sandi $\mathrm{C}$, et al. Neuroplastin-65 and a mimetic peptide derived from its homophilic binding site modulate neuritogenesis and neuronal plasticity. J Neurochem 2011, 117: 984-994.

3. Sarto-Jackson I, Milenkovic I, Smalla KH, Gundelfinger ED, Kaehne T, Herrera-Molina R, et al. The cell adhesion molecule neuroplastin-65 is a novel interaction partner of gammaaminobutyric acid type A receptors. J Biol Chem 2012, 287: 14201-14214.

4. Wilson MC, Kraus M, Marzban H, Sarna JR, Wang Y, Hawkes $\mathrm{R}$, et al. The neuroplastin adhesion molecules are accessory proteins that chaperone the monocarboxylate transporter MCT2 to the neuronal cell surface. PLoS One 2013, 8: e78654. 
5. Smalla KH, Matthies H, Langnase K, Shabir S, Bockers TM, Wyneken $\mathrm{U}$, et al. The synaptic glycoprotein neuroplastin is involved in long-term potentiation at hippocampal CA1 synapses. Proc Natl Acad Sci U S A 2000, 97: 4327-4332.

6. Herrera-Molina R, Sarto-Jackson I, Montenegro-Venegas C, Heine M, Smalla KH, Seidenbecher CI, et al. Structure of excitatory synapses and GABAA receptor localization at inhibitory synapses are regulated by neuroplastin-65. J Biol Chem 2014, 289: 8973-8988.

7. Desrivieres S, Lourdusamy A, Tao C, Toro R, Jia T, Loth E, et al. Single nucleotide polymorphism in the neuroplastin locus associates with cortical thickness and intellectual ability in adolescents. Mol Psychiatry 2015, 20: 263-274.

8. Saito A, Fujikura-Ouchi Y, Kuramasu A, Shimoda K, Akiyama $\mathrm{K}$, Matsuoka $\mathrm{H}$, et al. Association study of putative promoter polymorphisms in the neuroplastin gene and schizophrenia. Neurosci Lett 2007, 411: 168-173.

9. Bhattacharya S, Herrera-Molina R, Sabanov V, Ahmed T, Iscru $\mathrm{E}$, Stober F, et al. Genetically induced retrograde amnesia of associative memories after neuroplastin ablation. Biol Psychiatry 2017, 81: 124-135.

10. Carrott L, Bowl MR, Aguilar C, Johnson SL, Chessum L, West $\mathrm{M}$, et al. Absence of neuroplastin-65 affects synaptogenesis in mouse inner hair cells and causes profound hearing loss. J Neurosci 2016, 36: 222-234.

11. Zeng WZ, Grillet N, Dewey JB, Trouillet A, Krey JF, BarrGillespie PG, et al. Neuroplastin isoform Np55 is expressed in the stereocilia of outer hair cells and required for normal outer hair cell function. J Neurosci 2016, 36: 9201-9216.

12. Amuti S, Tang Y, Wu S, Liu L, Huang L, Zhang H, et al. Neuroplastin 65 mediates cognitive functions via excitatory/ inhibitory synapse imbalance and ERK signal pathway. Neurobiol Learn Mem 2016, 127: 72-83.

13. Dong S, Li C, Wu P, Tsien JZ, Hu Y. Environment enrichment rescues the neurodegenerative phenotypes in presenilins-deficient mice. Eur J Neurosci 2007, 26: 101-112.

14. Rajeevan MS, Ranamukhaarachchi DG, Vernon SD, Unger ER. Use of real-time quantitative PCR to validate the results of cDNA array and differential display PCR technologies. Methods 2001, 25: 443-451.

15. Ye J, Coulouris G, Zaretskaya I, Cutcutache I, Rozen S, Madden TL. Primer-BLAST: a tool to design target-specific primers for polymerase chain reaction. BMC Bioinformatics 2012, 13: 134.
16. van Amerongen $\mathrm{R}$, Nusse R. Towards an integrated view of Wnt signaling in development. Development 2009, 136: 3205-3214.

17. Owczarek S, Berezin V. Neuroplastin: cell adhesion molecule and signaling receptor. Int J Biochem Cell Biol 2012, 44: 1-5.

18. Empson RM, Buckby LE, Kraus M, Bates KJ, Crompton MR, Gundelfinger ED, et al. The cell adhesion molecule neuroplastin65 inhibits hippocampal long-term potentiation via a mitogenactivated protein kinase p38-dependent reduction in surface expression of GluR1-containing glutamate receptors. J Neurochem 2006, 99: 850-860.

19. Hussaini SM, Choi CI, Cho CH, Kim HJ, Jun H, Jang MH. Wnt signaling in neuropsychiatric disorders: ties with adult hippocampal neurogenesis and behavior. Neurosci Biobehav Rev 2014, 47: 369-383.

20. Kreutz MR, Langnaese K, Dieterich DC, Seidenbecher CI, Zuschratter W, Beesley PW, et al. Distribution of transcript and protein isoforms of the synaptic glycoprotein neuroplastin in rat retina. Invest Ophthalmol Vis Sci 2001, 42: 1907-1914.

21. McEntee WJ, Crook TH. Serotonin, memory, and the aging brain. Psychopharmacology (Berl) 1991, 103: 143-149.

22. Ma G, Fan H, Shen C, Wang W. Genetic and neuroimaging features of personality disorders: State of the art. Neurosci Bull 2016, 32: 286-306.

23. Rahimian R, Fakhfouri G, Ejtemaei Mehr S, Ghia JE, Genazzani AA, Payandemehr B, et al. Tropisetron attenuates amyloid-betainduced inflammatory and apoptotic responses in rats. Eur J Clin Invest 2013, 43: 1039-1051.

24. Gordon JA, Hen R. The serotonergic system and anxiety. Neuromolecular Med 2004, 5: 27-40.

25. Mosienko V, Bert B, Beis D, Matthes S, Fink H, Bader M, et al. Exaggerated aggression and decreased anxiety in mice deficient in brain serotonin. Transl Psychiatry 2012, 2: e122.

26. Sinhababu AK, Borchardt RT. Molecular mechanism of biological action of the serotonergic neurotoxin 5,7-dihydroxytryptamine. Neurochem Int 1988, 12: 273-284.

27. Dai JX, Han HL, Tian M, Cao J, Xiu JB, Song NN, et al. Enhanced contextual fear memory in central serotonin-deficient mice. Proc Natl Acad Sci U S A 2008, 105: 11981-11986.

28. Kelley SP, Bratt AM, Hodge CW. Targeted gene deletion of the 5-HT3A receptor subunit produces an anxiolytic phenotype in mice. Eur J Pharmacol 2003, 461: 19-25. 